\title{
Anne-Marie Châtelet, Franck Storne (dir.), Des Beaux-Arts à l'Université. Enseigner l'architecture à Strasbourg
}

Paris/Strasbourg, coédition Recherches/École nationale supérieure d'architecture de Strasbourg, volume I, Histoire et mémoires / volume II, Dessins, 2013.

\section{(2) OpenEdition}

\section{Journals}

Édition électronique

URL : http://journals.openedition.org/crau/431

DOI : $10.4000 /$ crau. 431

ISSN : 2547-5746

Éditeur

Éditions du patrimoine

Édition imprimée

Date de publication : 1 décembre 2014

Pagination : 247

ISBN : 978-2-7577-0379-3

ISSN : 1296-4077

Référence électronique

" Anne-Marie Châtelet, Franck Storne (dir.), Des Beaux-Arts à l'Université. Enseigner l'architecture à

Strasbourg ", Les Cahiers de la recherche architecturale et urbaine [En ligne], 30/31 | 2014, mis en ligne le 14 septembre 2017, consulté le 24 septembre 2020. URL : http://journals.openedition.org/crau/431 DOI : https://doi.org/10.4000/crau.431 


\section{Anne-Marie Châtelet, Franck Storne (dir.), Des Beaux-Arts à I'Université. Enseigner l'architecture à Strasbourg}

Paris / Strasbourg, co-édition Recherches / École nationale supérieure d'architecture de Strasbourg, volume I, Histoire et mémoires / volume II, Dessins, 2013, 584 p., 49 euros.

À l'automne 2013, la médiatique inauguration de l'extension de l'école d'architecture de Strasbourg par l'architecte Marc Mimram fut accompagnée d'un événement à l'écho plus discret : la publication de l'ouvrage Des Beaux-Arts à I'Université. Enseigner l'architecture à Strasbourg. Sous la direction scientifique d'Anne-Marie Châtelet et Franck Storne, les deux volumes publiés (vol. 1, Histoire et Mémoires ; vol. 2, Dessins) révèlent la richesse d'un fond archivistique et éclairent l'histoire de l'enseignement et de l'architecture dans l'est de la France des années 1921 à 2000.

Ce texte offre une contribution de première importance à l'histoire quasi inexistante des écoles régionales d'architecture $^{1}$. À la récurrente question Quel enseignement pour l'architecture ? la vingtaine de contributeurs mobilisés - 'étant pas tous liés à l'école, il faut bien le préciser - esquisse une réponse qui s'étire sur près d'un siècle offrant, loin d'une introspection égotique ou régionaliste, des éclairages sur les débats nationaux et la progressive décentralisation de l'enseignement.

La partie Histoire décline trois sections: "L'école en trois temps », "Lieux et enseignements", "Architectes en Alsace ». La première présente trois moments fondateurs : la création de l'école régionale jusqu'aux tumultes des années 1960, la refondation de 1965 à 1975, puis l'indépendance de 1975 à 2000. Les deuxième et troisième parties proposent plusieurs essais thématiques sur les espaces historiques de l'école, son fonctionnement et les aspects matériels, mais aussi le rapport avec d'autres établissements où l'on enseignait l'architecture et le milieu professionnel en Alsace. La partie Mémoires livre les réflexions et les combats de quelques élèves, enseignants et directeurs. C'est l'histoire de plusieurs conquêtes qui sont ici retracées dans un aller-retour permanent entre les problématiques locales et nationales. Si l'école est d'abord un outil de rayonnement identitaire à la suite du premier conflit mondial, sa progressive autonomie lui fait accepter et valoriser l'identité binationale de sa culture architecturale.

\section{L'école comme instrument de reconquête culturelle régionale}

À la différence de ses semblables, cette école régionale " $d^{\prime}$ extrême frontière ${ }^{2}$ " créée en 1921 sur décision de l'État a d'abord pour mission de renforcer et
1. À l'exception de l'article de Jean-Henri Fabre, "L'enseignement de l'architecture à Toulouse dans les années 1960 », Toulouse 45/75,

Toulouse, Nouvelles Éditions Loubatières, 2009, p. 250-299, et Daniel Le Couédic, "Genèse et premier âge d'une école d'architecture : Rennes 1909-1941 ", Arts de l'Ouest, Rennes, Presses universitaires de Rennes, 1995, p. 49-60. À noter également la journée d'études « Les écoles régionales d'architecture. Bilan et perspectives de recherches » qui s'est tenue à Strasbourg le 16 janvier 2014.
2. Rapport sur l'école régionale d'architecture à Strasbourg, 9 juin 1938 (archive de l'École nationale supérieure d'architecture de Strasbourg). 
de diffuser l'identité française sur le territoire de l'Alsace-Lorraine. MarieJeanne Dumont revient sur le fonctionnement de l'École nationale supérieure des beaux-arts (Ensba) et l'implication des groupes régionaux et des associations provinciales dans la création des écoles régionales à partir de 1904 . On y découvre le rôle de l'Alsace dans la politique publique régionaliste des années d'après-guerre et la détermination du groupe Rhin-Moselle de la SADG, Société des architectes diplômés par le gouvernement, au moment de la création de l'École régionale d'architecture de Strasbourg (Éras). Son histoire commence en 1921. Anne-Marie Châtelet retrace les conditions de sa création sous la direction de Robert Danis, grand défenseur de I'architecture française. Des controverses à l'inauguration dans le palais du Rhin face à l'université, elle analyse les circonstances matérielles et économiques de I'installation, puis la difficile évolution des effectifs et la nature du cursus (ateliers, enseignements et concours) empreint d'une dépendance idéologique et institutionnelle face à la maison mère parisienne. Après les années de guerre, la réouverture de l'Éras en 1945 marque un changement de direction avec un acteur majeur de la Reconstruction, Gustave Stoskopf, qui défendra la décentralisation de l'enseignement. La quête de reconnaissance de l'Eras s'intensifie et les retombées de la création de l'Ordre des architectes renforcent les velléités d'autonomie fragilisant l'autorité du quai Malaquais qui voit progressivement son élitisme et son centralisme menacés.

\section{Autonomie pédagogique et spatiale} La décennie 1965 -1975 correspond à la refondation. Michel Denès revient sur les prémisses de la dissolution de la section architecture aux Beaux-Arts et les turbulences des années 1968 qui précipitent la fin des ateliers tels qu'ils étaient pratiqués jusqu'alors. À Strasbourg, les accents contre-culturels s'impriment dans la courte publication Vroustch (1971-1972) réalisée par quelques étudiants de l'école. Les hésitations et expérimentations pédagogiques ainsi que les grèves de l'hiver 1974-1975 marquent la fin d'une époque où se fait jour le souhait d'un rattachement à l'université, la mise en place d'un secteur recherche et le projet de création d'agences d'architecture liées à l'enseignement. Si le rattachement de l'école d'architecture de Strasbourg à l'université fut acté en avril 2013, Françoise OlivierUtard rend compte de l'apport des universitaires strasbourgeois dans la formation de l'Éras.

Les années 1975 à 2000 sont celles de I'indépendance. Diego Peverelli les illustre avec des extraits de programmes pédagogiques (notamment celui de 1974-1975) et des comptes rendus de débats sur les orientations de l'enseignement de l'Unité pédagogique d'architecture (Upa) de Strasbourg. II livre une analyse minutieuse des contenus des programmes et de leurs évolutions évoquant les diverses postures et thématiques représentatives de l'enseignement du projet et des disciplines associées dans les différents cycles. À la conquête de l'autonomie pédagogique correspond celle d'une souveraineté spatiale. Le passage de l'incommode palais wilhelmien jusqu'à l'acquisition en 1981 d'un garage BMW désaffecté est retracé par Marion Freymann. Diego Peverelli explicite la reconversion de ce bâtiment commercial et industriel des années 1930 par les architectes Guy Clapot et Michel Moretti en phase avec la posture du retour à la ville de la fin des années 1970. Barbara Morovich rend compte de l'installation des étudiants et de l'ensemble du personnel administratif et enseignant dans cet espace de production, observant sous le prisme de la socio-anthropologie l'appropriation des espaces et les détournements. Mais au-delà de l'espace de cette nouvelle école, juste en face de la gare, c'est aussi une période où se structurent l'ouverture et les échanges dans l'espace rhénan.

\section{Vers la valorisation}

\section{d'une identité binationale}

L'identité binationale de Strasbourg ne cessera d'être questionnée dès le début de l'Éras, dans la formation même des étudiants à l'art de construire ; la différence avec l'Allemagne se veut marquée. Christiane Weber revient sur la spécificité de I'enseignement dispensé à l'École 
impériale technique de Strasbourg (fondée par les Allemands en 1895), laquelle a survécu aux annexions et aux conflits. En 1962, la possibilité d'une fusion avec l'Éras est vite abandonnée et aujourd'hui la formation d'architecte à I'Institut national de sciences appliquées (Insa) est l'un des reflets de ce bi-culturalisme. Matteo Porrino poursuit I'analyse de l'enseignement de la construction dans l'entre-deux-guerres à partir de la comparaison de deux carnets de notes inédits l'un de l'école régionale, l'autre de l'École nationale technique de Strasbourg (anciennement École impériale technique). À l'Éras, l'art de bâtir au-delà de la bonne technique était enrichi d'une dimension théorique questionnant la solution architecturale et la nature même de l'acte de projeter. Ce constat permet à l'auteur d'évoquer, par ailleurs, la séparation qui règne aujourd'hui entre les cours de sciences et techniques de la construction et les cours de projet. La composition du fonds de la bibliothèque et ses évolutions qu'analyse Franck Storne sont aussi révélatrices de cette identité contrariée.

Dès le début des années 1970, la dimension transfrontalière s'impose, l'encrage européen passe par le renforcement des liens de l'école avec d'autres en Allemagne (Karlsruhe et Stuttgart) mais également en Suisse. On constate par ailleurs que le projet urbain occupe très tôt une place centrale ${ }^{3}$, notamment autour de la figure enseignante de René Tabou- ret. Cette dimension est notamment un axe fort de la recherche dès la création de l'association pour la recherche près de I'Institut d'architecture et d'urbanisme (1976-1984). Cristiana Mazzoni, Andreea Grigorovschi et Luna d'Emilio en retracent les développements.

De la formation à la profession, c'est plus largement les évolutions du métier et les débats autour de l'identité régionale et patrimoniale qui marquent l'entre-deuxguerres. Nicolas Lefort questionne la réorganisation des services d'architecture d'Alsace-Lorraine après le retour à la France (1919-1939) et Denis Durand de Bousingen, le milieu architectural strasbourgeois (1910-1940).

\section{Des figures méconnues : portraits et témoignages}

Cet ouvrage éclaire des figures tutélaires méconnues tel Pierre Valentin Boudhors (1754-1831) qui fut l'instigateur de l'introduction du néoclassicisme en Alsace dont Véronique Umbrecht retrace le parcours et I'influence. Robert Danis (1879-1949) et Charles-Gustave Stoskopf (1907-2004) marquent également fortement la période. Claire Johann revient sur le premier, directeur-fondateur de l'Éras, grand défenseur de l'architecture française, qui a œuvré au service du patrimoine et participé à la fondation du corps des architectes des bâtiments de France. Gauthier Bolle analyse la carrière étonnante de CharlesGustave Stoskopf, deuxième directeur de l'école dont l'attachement régionaliste ne l'empêchera pas de devenir une figure majeure de la Reconstruction, nationalement reconnue 4 .

De ces plus illustres acteurs aux étudiants anonymes, l'histoire collective n'a pas omis de questionner les seconds. Ce sont les étudiantes de l'Éras dans l'entre-deuxguerres dont Amandine Diener retrace le parcours et la difficile reconnaissance. Le cas de ces quelques femmes architectes courageusement élèves à une époque où leur capacité à exercer le métier était sérieusement questionnée dépasse, là aussi, le cadre strasbourgeois.

La partie Mémoires donne ensuite à lire des témoignages d'anciennes élèves et enseignants des années 1920 à 2000. Le volume ॥ Dessins complète cette lecture offrant - en pleine page - une sélection de projets : d'admission, de seconde et première classe, puis les diplômes et prix de Rome, et enfin ceux d'après 1968. Ces plus de deux cents travaux d'archives sélectionnés et collectés composent un précieux panel de la production strasbourgeoise.

Les directeurs exposent également leurs combats. De l'amertume de Gustave Stoskopf aux plus enthousiastes contributions d'Étienne de Cointet et d'Yves Ayrault succèdent deux points de vue éclairant sur l'enseignement ceux de Gustave Stoskopf (1956) et de Jean-Pierre Halévy (1970). Ils font revivre la longue bataille de la province pour l'autonomie et la nécessité d'une politique clairement affirmée en sa faveur, appelant de leurs

4. Gauthier Bolle, Gustave Stoskopf (1907-2004), architecte et poète alsacien. De la Reconstruction aux grands ensembles, thèse en cours de finalisation sous la direction d'Anne-Marie Châtelet, université de Strasbourg / École nationale supérieure d'architecture de Strasbourg. co-organise notamment le premier colloque international, en 1981, consacré au projet urbain avec le Conseil de l'Europe, la ville de Strasbourg et l'Agence

d'urbanisme : "Le projet urbain dans I

a construction de la cité ». 
vœux la réalisation d'une pédagogie et d'une culture architecturale propre qui fassent de chaque école un laboratoire de pensée.

Les fragments ici rassemblés font portrait, renforçant l'identité de l'une des vingt écoles nationales supérieures d'architecture. Cet ouvrage en appelle d'autres, composants actifs et réflexifs, nécessaires à la pensée de l'enseignement de l'architecture du $x x^{e}$ siècle.

FANNY LOPEZ

Docteur en histoire de l'architecture, enseignante titulaire à l'école nationale supérieure d'architecture de Strasbourg, chercheuse au LIAT (Laboratoire infrastructure architecture territoire) à l'école nationale supérieure d'architecture Paris-Malaquais. 